\title{
Multidisciplinary approach to oesophageal cancer
}

\author{
Fernando López
}

$\mathrm{O}^{\mathrm{s}}$ esophageal cancer has one of the highest mortality rates amongst patients with gastrointestinal cancers. Five-year survival is less than $20 \%$ in most series. Only patients diagnosed in an early stage can be cured. The incidence of oesophageal and gastroesophageal junction adenocarcinoma has been rising during recent years and has now become the most frequent tumour type in the West. Across Europe, 45,000 new cases per year, 40,000 deaths per year and an overall 5-year survival of $15 \%$ have been estimated. In recent decades there has been a significant improvement in the management of oesophageal cancer. This includes better patient selection, refined surgical technique, improvement in perioperative care and a more rational application of adjuvant therapies. Modern management of patients with oesophageal cancer requires a multidisciplinary approach involving surgeons, medical oncologists, gastroenterologists, radiologists, radiation oncologists and pathologists.

Patients with oesophageal cancer can be divided into two main groups. The first group of patients are diagnosed in an asymptomatic or an early stage (<T2-N0). In these patients an almost $90 \%$ 5-year survival rate can be achieved [1]. Surgery with no additional therapies is the main option for these patients. In very early stages endoscopic mucosal resection can be performed, especially for squamous cell cancer confined to the lamina propria, although results of this treatment are still unclear [2]. Unfortunately this is not very common in Western countries. The second group of patients are diagnosed in an advanced stage. It is in these cases that accurate staging has a really important role. Endoscopic ultrasonography (EUS) is regarded as the best staging modality for $\mathrm{T}$ and $\mathrm{N}$ stage. The accuracy of determining $\mathrm{T}$ stage ranges between 85 and $90 \%$ and $\mathrm{N}$

F. López (ه)

Upper Gastrointestinal Surgery Unit Institute of Health Research INCLIVA

Hospital Clinic of Valencia

Valencia, Spain

e-mail: ferlomo@gmail.com stage ranges between 70 and 90\% [3, 4]. For detection of distant metastasis or T4 disease, computerised tomography (CT) scans have a major role, although their sensitivity for detection of metastasis depends on the size of the lesions and less than $50 \%$ are detected when smaller than $1 \mathrm{~cm}$. Positron emission tomography (PET) scans are becoming popular for the detection of distant metastasis and for evaluating response to chemotherapy, although their sensitivity for detection of regional lymph nodes is still lower than that of EUS. Nowadays, a CT scan is complimentary to a PET scan when anatomical enlargement of nodes is correlated with metabolic activity. Bronchoscopy is also mandatory in those tumours localised in the mid and upper third of the oesophagus. Invasion of the bronchi or trachea is a formal contraindication for surgery. Some groups also include an ultrasonography of the neck and fine needle aspiration for the detection of cervical nodal metastasis in their diagnostic algorithm [5]. The role of staging laparoscopy is still unclear. Its use is limited to cases of gastroesophageal junction adenocarcinoma with enlarged abdominal lymph nodes in the CT scan.

It is very important to exclude the presence of metastasis in these patients since the therapeutic strategy will be based on this. For all these reasons, collaboration between radiologists, endoscopists and gastroenterologists is crucial for optimal staging. Despite all of these efforts, there are a subgroup of patients who develop distant metastasis in a short period after treatment, which means that these patients had metastatic disease at the time of the instauration of treatment. These cases are especially disappointing for the medical team.

Optimal patient selection is also very important. Surgery is only justified when a low morbidity and mortality rate can be achieved and when a reasonable prognosis is expected. A R0 resection with adequate margins is considered as one of the most important prognostic factors. Patient's physiological reserve must also be assessed. Most of the time this is based on the surgeons' experience. There are some objective scores that can help to assess operative risk and therefore patient selection [6]. Furthermore, cardiopulmonary, renal and liver function must be assessed in all cases. Performance and nutritional status are also important as they have been associated with postoperative 
morbidity. Oesophagectomy outcome can be improved when performed in high-volume centres. In these cases the mortality rate reported is $2-3 \%$ compared to $13-20 \%$ when oesophagectomy is performed by inexperienced surgeons $[7,8]$. Authorities have an important role in the regulation of which centres are designated to perform this type of surgery. In the UK gastroesophageal surgery is now concentrated in 23 centres across the country.

Controversy about the transthoracic or transhiatal approaches remain unsolved. Moreover, a variety of minimally invasive surgery (MIS) techniques have recently appeared with different combinations of thoracoscopy and laparoscopy. Although the most popular is thoracoscopic oesophageal resection and laparotomy, this depends on the surgeon's preferences. A new intergroup trial (ECOG 2202) to assess MIS is underway [9]. Another surgical controversy is the extension of the lymphadenectomy. Three-field lymphadenectomy seems to be associated with a better survival according to the Japanese experience, although this is correlated with higher morbidity rates. Two-field lymphadenectomy is recommended for tumours localised in the mid and lower thirds of the oesophagus and neck lymph node dissection is reserved for tumours in the upper third.

However, without any doubt, multimodality treatment is the choice for patients with advanced oesophageal cancer. This combines different regimens of chemotherapy, radiotherapy and surgery, with great variation between countries. In Europe perioperative chemotherapy following surgery is often practised, whereas in the USA preoperative chemoradiation is widely accepted. In Asia, surgery remains the main curative option for oesophageal cancer. Even chemoradiation alone has been reported to show similar results to surgery in terms of survival. Thus this strategy is now reserved for squamous cell carcinoma tumour type localised in the upper third or those patients with high surgical risk.

In an attempt to clarify the role of chemotherapy/radiotherapy, two important trials have been reported. The UK MRC OEO2 study compares preoperative chemotherapy (5-FU/cisplatin) following surgery with surgery alone. This study demonstrated a superior survival in the chemotherapy and surgery group, but similar results were not found by the American Intergroup 0113 study, which had a very similar design. The Intergroup 0116 study compared surgery following chemoradiation with surgery alone and concluded higher survival rates in the chemoradiation group. However, this study has been widely criticised for the poor quality of the surgery performed. Most of the patients included in the British trial $(65 \%)$ and all of the patients of the Intergroup 0116 trial had adenocarcinoma. In the Intergroup 0113 trial tumour types were well balanced between squamous cell carcinoma and adenocarcinoma (47\% vs. $53 \%)$.

An updated Cochrane systematic review covering the role of preoperative chemotherapy has recently been published. This included 12 randomised trials involving 2097 patients and the conclusion was that preoperative chemotherapy plus surgery may offer a survival advantage compared to surgery alone for resectable thoracic oesophageal cancer, but the evidence is inconclusive [10].

Selection of the best multimodality combination remains a challenge. Collaboration between multidisciplinary experts can help to individualise treatments among patients with oesophageal cancer. The optimal sequence of integrating surgery, chemotherapy and radiotherapy will be addressed in ongoing randomised trials. In this issue of Clinical and Translational Oncology, Valentí et al. provide a good practical example of this multimodality approach [11].

Improvements have also been made in palliative patients. The most frequent symptom presented in these patients is dysphagia. The development of self-expanding stents has contributed significantly to the management of palliative patients hence surgery is now reserved for special situations.

Targeted therapy is under development. New therapeutic agents such as monoclonal antibodies, tyrosine-kinase inhibitors, matrix metalloproteinase inhibitors and cycloxygenase inhibitors have been employed without success for the moment.

In summary, the current evidence favours a multidisciplinary approach to oesophageal cancer. Advancement of technology allows more accurate staging and provides guidance on patient selection for different therapeutic options. Treatment of patients should be individualised and this can only be achieved by a close collaboration between different specialists.

\section{References}

1. Wang GQ, Jiao GG, Chang FB et al (2004) Longterm results of operation for 420 patients with early squamous cell esophageal carcinoma discovered by screening. Ann Thorac Surg 77:17401744

2. Soetikno R, Kaltenbach T, Yeh R et al (2005) Endoscopic mucosal resection for early cancers of the upper gastrointestinal tract. J Clin Oncol 23:4490-4498

3. Rösch T (1995) Endosonographic staging of esophageal cancer: a review of literature results. Gastrointest Endosc Clin N Am 5:537-547
4. Reed CE, Mishra G, Sahai AV et al (1999) Esophageal cancer staging: improved accuracy by endoscopic ultrasound of celiac lymph nodes. Ann Thorac Surg 67:319-321

5. Law S (2010) Esophagectomy without mortality: what can surgeons do? J Gastrointest Surg 14[Suppl 1]:S101-107

6. Ferguson MK, Celauro AD, Prachand V (2011) Prediction of major pulmonary complications after esophagectomy. Ann Thorac Surg 91:14941500

7. Birkmeyer JD, Siewers AE, Finlayson EV et al (2002) Hospital volume and surgical mortality in the United States. N Engl J Med 346:11281137
8. Birkmeyer JD, Stukel TA, Siewers AE et al (2003) Surgeon volume and operative mortality in the United States. N Engl J Med 349:2117-2127

9. Luketich JD, Alvelo-Rivera M, Buenaventura PO et al (2003) Minimally invasive esophagectomy: outcomes in 222 patients. Ann Surg 238:486-494

10. Vogt K, Fenlon D, Rhodes S, Malthaner R (2010) Preoperative chemotherapy for resectable thoracic esophageal cancer. Cochrane Datab Syst Rev (3):CD001556

11. Valentí V, Hernandez-Lizoain JL, Martinez-Regueira F et al (2011) Transthoracic oesophagectomy with lymphadenectomy in 100 oesophageal cancer patients. Multidisciplinary approach. Clin Transl Oncol 13:899-903 